\title{
Usefulness of an Overtube Device in Gastrointestinal Endoscopy
}

\author{
Seung Han Kim \\ Division of Gastroenterology and Hepatology, Department of Internal Medicine, Institute of Gastrointestinal Medical Instrument Research, \\ Korea University College of Medicine, Seoul, Korea
}

See "Technical Feasibility of a Guidetube for Various Endoscopic Procedures in Human Gastrointestinal Simulators" by Dong Seok Lee, Byeong Gwan Kim, Kook Lae Lee, et al., on page 247-251.

An overtube is a sleeve-like equipment made of semi-rigid plastic, designed to assist endoscopy. ${ }^{1}$ An overtube of a larger diameter than that of an endoscope is needed for providing a route through the gastrointestinal (GI) tract. Overtubes were created to prevent aspiration events or mucosal laceration of the GI tract during the endoscopic procedure. They can be used for overcoming obstacles such as difficult anatomical structures and limited insertion depth and for facilitating the process of repeated insertion and withdrawal.

An overtube has proximal closure and a tapered soft distal tip. The anal side edge of the device needs to be atraumatic, and it has an inner width that matches the diameter of the endoscope. The length and diameter of overtubes differ to accommodate the intended application as well as the approach route.

Although an overtube has the advantages of facilitating endoscopic procedures, it also has inevitable safety issues. The most commonly reported complications involving the use of overtubes are mucosal scratches or tears. These side effects mostly occur because of the large diameter and mucosal pinching between the endoscope and overtube. ${ }^{2,3}$ Other serious complications include pharyngeal or esophageal perfora-

Received: April 12, 2019 Accepted: May 8, 2019

Correspondence: Seung Han Kim

Division of Gastroenterology and Hepatology, Department of Internal Medicine, Korea University College of Medicine, 73 Inchon-ro, Seongbuk-gu, Seoul 02841, Korea

Tel: +82-2-920-6555, Fax: +82-2-920-1943, E-mail: kimseunghan09@gmail.com ORCID: https://orcid.org/0000-0001-9247-9175

(cc) This is an Open Access article distributed under the terms of the Creative Commons Attribution Non-Commercial License (http://creativecommons.org/ licenses/by-nc/3.0) which permits unrestricted non-commercial use, distribution, and reproduction in any medium, provided the original work is properly cited. tion, rupture of varices, pneumomediastinum, and temporary vocal cord paralysis. ${ }^{4-6}$

In the clinical setting, an overtube is commonly used in the removal of foreign material, esophageal variceal band ligation using separately attached bands, assisting colonoscope insertion, and endoscopic procedures, such as endoscopic submucosal dissection. ${ }^{7-9}$ During endoscopic procedures, the overtube prevents mucosal damage and tracheal aspiration and provides an easy route for repeated endoscopic insertion.

In the current issue, Lee et al. investigated the technical feasibility of a newly developed endoscopic overtube for various endoscopic procedures in a GI simulator. ${ }^{10}$ This new overtube had inner and outer tubes. Silicone was used as the outer tube material to reduce mucosal damage. A total of 15 endoscopic procedures were performed using the GI simulator. The endoscope insertion time using the guide-tube was reduced by approximately $70 \%$. Moreover, the driving force of the developed guide-tube was smaller than that of a simple overtube (proximal esophagus: $1.5 \pm 0.1 \mathrm{~kg}$ vs. $4.4 \pm 0.1 \mathrm{~kg}$ ). The mean retrieval time for a 2-cm polyp with the guide-tube was significantly decreased compared to that of using a conventional procedure.

The current study showed that endoscopic procedures assisted by the newly developed overtube were fast and safe in the GI simulator but not in the human GI tract. Although the authors presented that guide-tube-assisted procedures were superior in terms of speed, convenience, safety, and price, they did not directly compare the newly developed overtube with conventional overtube systems. In addition, because it was not applied in the human GI tract, there are no data regarding the efficacy and possible complications of the newly developed 
overtube. We should also consider the cost-effectiveness of the newly developed overtube because previously described insertion techniques can reduce the risks during overtube insertion with conventional overtubes.

An overtube can be practically used in clinical endoscopic procedures. With its capability to ease the luminal approach, it plays an important role in expanding endoscopic surgical techniques. There are several overtube systems that are being improved. Products that overcome various drawbacks are continuously being developed and marketed. The overtube used in this study has the potential to reduce complications during the procedure, can be used in various indications, and is attractive in terms of price. However, its efficacy was evaluated only in the simulator and not in the human GI tract. Moreover, it was not compared with conventional overtubes. Therefore, we cannot sufficiently conclude that the new overtube can overcome the limitations of conventional overtubes and be used with minimal complications in the human GI tract. To address this issue, the authors must compare the newly developed overtube with conventional overtubes in the human GI tract.

\section{Conflicts of Interest}

The author has no financial conflicts of interest.

\section{Acknowledgments}

This work was supported by the National Research Foundation of Korea (NRF) grant funded by the Korean Government (NRF-2019R1C1C1009819) and by the Ministry of Trade, Industry \& Energy (MOTIE, Korea) under Industrial Technology Innovation Program No. 10060251, "Development of diagnostic device for functional dyspepsia based on Korean-Western medicine fusion abdominal diagnosis".

\section{REFERENCES}

1. ASGE Technology Committee, Tierney WM, Adler DG, et al. Overtube use in gastrointestinal endoscopy. Gastrointest Endosc 2009;70:828-834.

2. Holderman WH, Etzkorn KP, Patel SA, Harig JM, Watkins JL. Endoscopic findings and overtube-related complications associated with esophageal variceal ligation. J Clin Gastroenterol 1995;21:91-94.

3. Berkelhammer C, Madhav G, Lyon S, Roberts J. "Pinch" injury during overtube placement in upper endoscopy. Gastrointest Endosc 1993;39:186-188

4. Jeffries MA, Scheiman JM. Overtube separation. Gastrointest Endosc 1998;47:435-436.

5. el-Newihi HM, Mihas AA. Esophageal perforation as a complication of endoscopic overtube insertion. Am J Gastroenterol 1994;89:953-954.

6. Dinning JP, Jaffe PE. Delayed presentation of esophageal perforation as a result of overtube placement. J Clin Gastroenterol 1997;24:250-252.

7. Shin JS, Sheu BS, Lin XZ, Chen CY, Leow TC. Longitudinal linear-incised overtube for endoscopic removal of larger ingested foreign bodies. Gastrointest Endosc 1995;41:618.

8. Stiegmann GV, Goff JS, Michaletz-Onody PA, et al. Endoscopic sclerotherapy as compared with endoscopic ligation for bleeding esophageal varices. N Engl J Med 1992;326:1527-1532.

9. Haber G, Whalen LK. A prospective study to evaluate the ShapeLock guide to enable complete colonoscopy in previously failed cases. Gastrointest Endosc 2006;63:AB226.

10. Lee DS, Kim BG, Lee KL, Jung YJ, Kim JW. Technical feasibility of a guidetube for various endoscopic procedures in human gastrointestinal simulators. Clin Endosc 2019;52:247-251. 